\title{
BER Analysis of OFDM Receiver Using MMSE Channel Estimation Method with PTS for Different Subblocks
}

\author{
M. Thanigasalam and P. Dananjayan
}

\begin{abstract}
Orthogonal frequency division multiplexing (OFDM) is widely used multi-carrier modulation technique in wireless networks because of its superiority. Partial Transmit Sequence (PTS) is one of the many methods of PAPR reduction technique that have been proposed to address the PAPR issue in OFDM systems. But PTS suffers from high computational complexity which increases exponentially with increase in the number of subblocks. Modified PTS technique has reduced this computational complexity by using neighborhood search algorithm to find the optimum set of phase factors to reduce the PAPR of the OFDM signal. In this work, MMSE channel estimation algorithm is used to evaluate the performance of the OFDM receiver using PTS as the PAPR reduction technique. The receiver performance is analyzed in terms of BER for different number of subblocks. Simulation results show that BER increases with PAPR reduction.
\end{abstract}

Index Terms - Bit error rate (BER), minimum mean square error (MMSE) channel estimation, peak-to-average power ratio (PAPR), partial transmit sequence (PTS).

\section{INTRODUCTION}

The demand for wireless communication system with high data rate and spectral efficiency has made Orthogonal Frequency Division Multiplexing (OFDM) to be the dominant and promising candidate in mobile communication. Over the years, OFDM has been implemented in various standards such as Digital Audio Broadcasting (DAB), Digital Video Broadcasting (DVB), and Wireless Local Area Network because of its tolerance towards multipath fading [1], [2]. The approach in OFDM is to split the high data rate sequence into $N$ symbol groups [3], so that each group consists of a sequence with lower symbol rate $1 / N$ than the original. The signal band is made up of $N$ orthogonal carrier waves and each one is modulated by different symbol groups. This way the symbol rate $W=1 / T$ on each carrier is reduced so that it is less than the coherence bandwidth of the channel. The introduction of guard band, called cyclic prefix (extension of the OFDM signal with its tail portion), avoids the inter-symbol interference (ISI), which if actually present will degrade the BER performance. The major disadvantage with OFDM is its high Peak-to-Average Power Ratio (PAPR) compared to a single carrier signal because an OFDM signal is the sum of many narrowband signals in time domain. The high PAPR imposes complexity in Digital-to-analog converter (DAC) and the High Power Amplifier (HPA). Both DAC and HPA must operate with a wider dynamic range for

Manuscript received April 3, 2013; revised June 18, 2013.

M. Thanigasalam and P. Dananjayan are with Department of Electronics and Communication Engineering, Karaikal Polytechnic College, Karaikal, Puducherry, India, (e-mail: thanigasalam1967@gmail.com, pdananjayan@pec.edu). prevention of spectral growth and out-of-band noise. The presence of high PAPR increases the cost of OFDM system. Many techniques to deal with PAPR issues have been proposed. These techniques include Clipping [4]-[7], Coding [8], [9], Multiple signal representation such as selective mapping (SLM) and PTS [10]-[13]. PTS is a distortionless method of PAPR reduction where the OFDM data block is divided into subblocks and each subblock is optimally shifted in phase, in order to reduce the probability of occurrence of high PAPR. The PAPR reduction is more with increase in the number of subblocks. But, the computational complexity of PTS scheme increases exponentially with increase in the number of subblocks, because of the increase in the number of $N$-dimensional IFFTs. In the literature, Modified PTS technique has been proposed to reduce the computational complexity. In Modified PTS technique, the optimal phase factor which reduces the PAPR of the OFDM signal is searched by using a neighborhood search algorithm by assuming a threshold value of PAPR. Channel estimation is the key technology of wireless receiver designs in OFDM systems. The transmitted signal undergoes reflections and scattering in the wireless channel before reaching the receiver. The signal reaches the receiver in multiple paths due to the mobility of the transmitters, receivers and scattering objects. The received signal is spread out in time, frequency and angle due to the detrimental effects of the wireless channel, thereby degrading the performance of the receiver. In OFDM receiver, the effect of the channel on the signal is estimated to increase the performance of the receiver. The Linear MMSE channel estimation algorithm is used to estimate the channel and to investigate the performance of the receiver for PAPR reduced signal using PTS as the PAPR reduction techniques.

\section{PAPR REDUCTION WITH PTS}

Consider an OFDM system with $N$ orthogonal subcarriers. Then, each symbol in a block consisting of $N$ symbols is modulated by one of the $N$ orthogonal subcarriers. Let the input block of symbols be $X_{d}(k)$ be

$$
X_{d}=\left[X_{d}(0), X_{d}(1), X_{d}(2), \ldots, X_{d}(N-1)\right]^{T}
$$

Each element of the vector shown in equation (1) represents an independently modulated data symbol. The OFDM signal is formed by adding all the modulated subcarriers. The subcarriers used are orthogonal such that the frequency of separation between adjacent subcarriers is 
$\Delta f=1 / T$, where $T$ is the duration of the OFDM signal.

The time domain representation of transmitted signal is given by,

$$
x(n)=\frac{1}{\sqrt{N}} \sum_{k=0}^{N-1} X_{d}(k) e^{\frac{j 2 \pi k n}{N}}, 0 \leq n \leq N-1 ; 0 \leq k \leq N-1
$$

The PAPR of the transmitted OFDM signal is defined as the ratio of the maximum power to the average power of the transmitted signal, which is given by,

$$
\operatorname{PAPR}(x)=10 \log _{10} \frac{\max _{0 \leq n \leq N-1}|x(n)|^{2}}{E\left[|x(n)|^{2}\right]}
$$

where $E[$.$] is the expectation operator. In the PTS method of$ PAPR reduction, the input data block consisting of $N$ symbols is partitioned into $V$ subblocks of equal size, represented as

$$
X_{d}=\left[X_{d}^{0}, X_{d}^{1}, X_{d}^{2}, \ldots, X_{d}^{V-1}\right]^{T}
$$

Each sub-block [14] is then multiplied by a corresponding phase factor $b_{v}=e^{j \varphi v}$ where $v=1,2,3, \ldots \ldots, V$. After IFFT operation, the time domain signal is given by

$$
\mathrm{x}=\operatorname{IFFT}\left\{\sum_{v=1}^{V} b_{v} X^{v}\right\}=\sum_{v=1}^{V} b_{v} \mathrm{x}^{v}
$$

where $x^{v}$ is partial transmitted sequence. The phase factor vector is chosen from an allowed set $b=\{+1,-1,+j,-j\}$ such that the PAPR can be minimized [13]. Then the time domain signal with lowest PAPR is given by

$$
\tilde{x}=\sum_{v=1}^{v} \tilde{b}_{v} x^{v}
$$

where $\tilde{b}_{v}$ is optimum set of phase factor which will produce a low PAPR OFDM signal. The computational complexity increases because of the search of optimum set of phase factors from the allowed set $b_{v}$. Therefore, with increase in the number of subblocks, the computational complexity increases exponentially. The distribution of PAPR in the OFDM signal has stochastic characteristics and so it is analyzed using complementary cumulative distribution function $(\mathrm{CCDF})$ which is defined as the probability that the PAPR of the OFDM signal exceeds a threshold value $P A P R_{0}$. Mathematically, CCDF is defined as,

$$
C C D F(P A P R(x(n)))=\operatorname{Pr}\left(\operatorname{PAPR}(x(n))>P A P R_{0}\right)
$$

Modified PTS is proposed to determine the optimum phase factor vector to reduce the computational complexity of PTS [14]. By using modified PTS algorithm, a better PAPR performance than PTS can be achieved but with reduced computational complexity.

\section{Minimum MEAN SQUARE ERROR (MMSE) CHANNEL ESTIMATION}

In wireless communications, to improve the receiver performance, it is required to estimate the channel before demodulation. The channel estimation techniques can be blind or non-blind. Pilot assisted channel estimation is a non-blind Channel estimation technique, where known symbols, called Pilots are embedded into certain sub-carriers of each OFDM symbol. The received signal at known pilot locations are then interpolated for estimating the complete channel. Blind channel estimation techniques [15] do not use pilots in order to achieve high spectral efficiency. Non-blind techniques are implemented with high computational complexity and require large amount of data to evaluate the statistics of the channel. In non-blind channel estimation, previous information about the channel is used. Data aided channel estimation is a type of non-blind technique, where a complete or a portion of the symbol which is known to the receiver is transmitted. If the known information is sent over one or more OFDM symbols, then channel estimation technique is said to be Training symbols based. If the known information is inserted into the data, then it is called pilot aided channel estimation [16]-[19]. Pilot aided channel estimation can have different structures based on the arrangement of pilot tones; block type, comb type and lattice type. The block type of pilot arrangement is suitable for frequency selective channels. Comb type pilot arrangement use pilot tones at periodically located subcarriers of every OFDM symbol. This type of pilot arrangement is used for estimation of the channel along the frequency axis. Comb-type pilot arrangement is suitable for fast-fading channels. Lattice type pilot arrangement permit the estimation of the channel in both time and frequency axis. Here the pilot tones are scattered in both time and frequency axis. The PAPR reduced OFDM signal when transmitted through the wireless channel undergoes distortion. The received signal is distorted because of the characteristics of the wireless radio channel. For better BER performance the channel is estimated and compensated at the receiver. In OFDM system, the orthogonal property of the subcarriers allows that each subcarrier to be identified as an independent channel. So the received signal is the product of the transmitted signal and frequency response of the channel [15].

$$
Y(k)=X(k) H(k)+W(k)
$$

where $Y(k)$ is the received signal, $X(k)$ is the transmitted signal, $H(k)$ is the frequency response of the channel and $W(k)$ is the additive white Gaussian noise.

The linear MMSE channel estimation algorithm is used to evaluate the BER performance of PAPR reduced OFDM signal using comb-type pilot arrangement. Let $P$ denote locations of the pilot tones in the OFDM symbol of $N$ subcarriers. 


$$
P=\left\{l_{0}, l_{1}, \ldots, l_{N_{P}-1}\right\}
$$

Let the pilot tones in the OFDM symbol be represented by the set $X_{p}=\left[X_{p}(0), X_{p}(1), \ldots, X_{p}(N-1)\right]$, where $N_{p}$ is the number of pilot tones. $X_{p}(k)$ is the pilot tone when $k$ is an element of $P$. Consider another set which is the complement of the set $P$, denoted by $P^{\prime}$ to represent the locations of the data symbols. Let the set $X_{d}=\left[X_{d}(0), X_{d}(1), \ldots, X_{d}(N-1)\right]$ represent the data symbols, where $X_{d}(k)$ represents the corresponding data symbol for $k$ an element in $P^{\prime}$. Now, the OFDM symbol is,

$$
\begin{aligned}
X(k) & =X_{d}(k)+X_{p}(k) \\
& =\left\{\begin{array}{c}
X_{d}(k), k \in P^{\prime} \\
X_{p}(k), k \in P
\end{array}\right\}
\end{aligned}
$$

After the FFT operation at the receiver, the received signal is $Y(k)=X(k) H(k)+W(k)$. Let $\hat{H}(k)$ be the channel estimate of $H(k)$. Then,

$$
\hat{H}(k)=\hat{H}_{d}(k)+\hat{H}_{p}(k)
$$

where $\hat{H}_{d}(k)$ is the channel frequency response corresponding to the data locations and $\hat{H}_{p}(k)$ is the estimated channel frequency response at the locations of pilot tones. The Linear MMSE of the channel [20] is given by,

$$
\hat{H}=R_{h h}\left[R_{h h}+\frac{I}{S N R}\right]^{-1} H_{p}(k)
$$

where $H_{p}(k)$ is the least squares (LS) estimate of $H$ at the locations of the pilot, $R_{h h}$ is the covariance matrix of the channel, $S N R$ is the signal-to- noise ratio and $I$ is the identity matrix. The locations of the pilot tones are known at the receiver. So the LS estimate of the channel frequency response at the locations of pilot can be determined by using

$$
\hat{H}_{p}(k)=\frac{Y(k)}{X_{p}(k)}
$$

The LS estimates at the pilot locations are interpolated to estimate the channel $\hat{H}_{d}(k)$ at the locations of the data symbols. Then the estimated data symbol $\hat{X}_{d}(k)$ is given by

$$
\hat{X}_{d}(k)=\frac{Y(k)}{\hat{H}_{d}(k)}
$$

The performance measure employed in frequency domain channel estimates is Mean square error (MSE) and it is defined by

$$
M S E=E\left[|H(k)-\hat{H}(k)|^{2}\right]
$$

BER performance measure is also used to evaluate the performance of the receiver using MMSE channel estimation method.

\section{RESUlTS AND DisCUSSION}

The performance of the receiver is analyzed using BER and MSE parameters for PAPR reduced OFDM signal with different number of subblocks, using MATLAB software. The simulation parameters considered for analysis are given in Table I [20].

TABLE I: SIMULATION PARAMETER FOR PTS

\begin{tabular}{ll}
\hline \hline Simulation parameters & Type value \\
\hline Number of subcarriers & 256 \\
Number of subblocks & $2,4,8$ and 16 \\
Guard band & 8 \\
Modulation scheme & 16 QAM \\
Phase factors & $+1,-1$ \\
\hline \hline
\end{tabular}

Fig. 1 shows the PAPR performance of OFDM signal without PTS and PTS for $N=256$ for different subblocks $V=$ 2, 4, 8 and 16 .

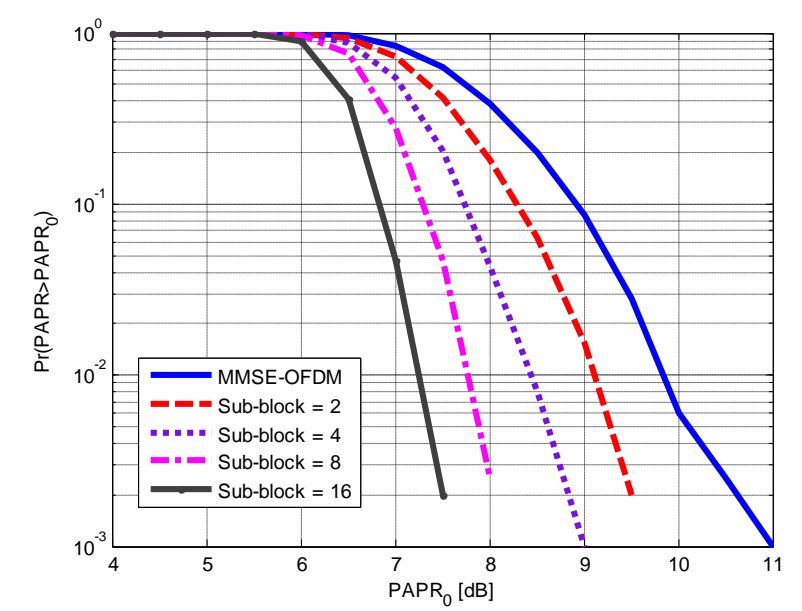

Fig. 1. PAPR performance of various subblocks $\mathrm{V}=2,4,8$ and 16 with $\mathrm{N}=$ 256.

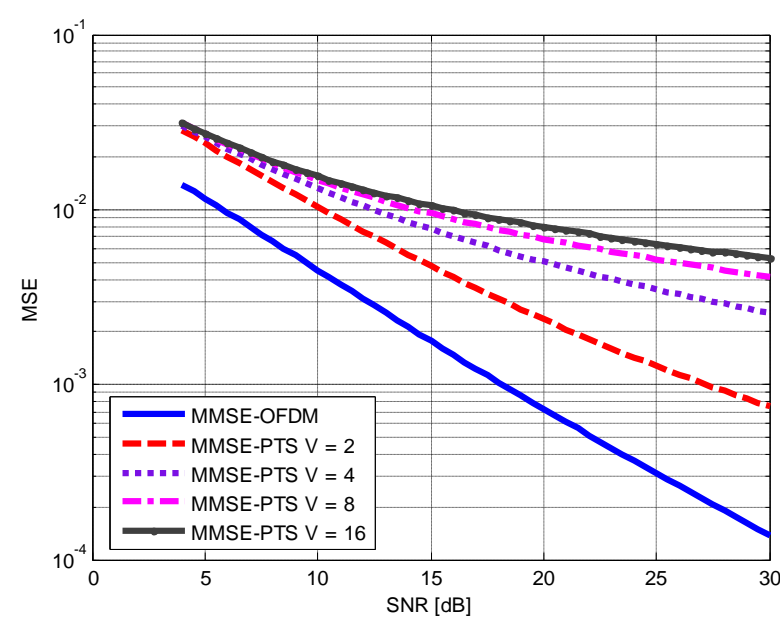

Fig. 2. Mean Square Error for MMSE estimator for different subblocks with $\mathrm{N}=256$ 
Fig. 2 depicts the mean square error of the channel versus SNR for the MMSE channel estimator, taking into account the channel statistics. Due to PAPR reduction, the MSE increases with increase in the number of sub-blocks.

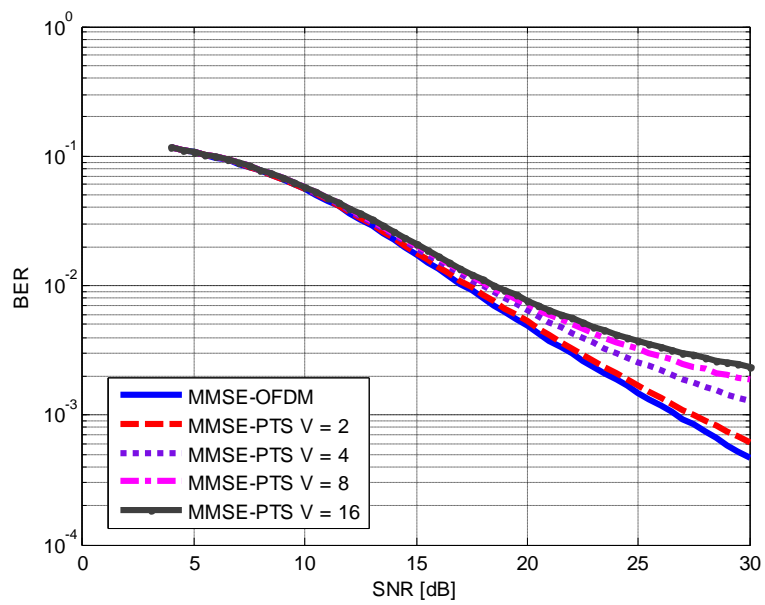

Fig. 3. BER for MMSE estimator for different subblocks $V=2,4,8$ and 16 with $\mathrm{N}=256$.

Fig. 3 shows the BER performance of the receiver with MMSE as the channel estimation technique. The bit-error rate decreases with increase in the number of subblocks, due to reduction in PAPR with increase in the number of subblocks. The BER performance is better than LS channel estimator [21].

\section{CONCLUSION}

In this paper, the MMSE channel estimation technique is used to analyze the performance of PAPR reduced OFDM signal using PTS as the PAPR reduction technique. Because of PAPR reduction, there is a slight degradation in the BER performance of the receiver. Also, the BER performance is improved by using MMSE channel estimation technique.

\section{REFERENCES}

[1] R. Van Nee, G. Awater, M. Morikura, H. Takanashi, M. Webster, and K. W. Halford, "New high-rate wireless LAN standards," IEEE Communications Magazine, vol. 37, pp. 82-88, Dec. 1999.

[2] L. J. Cimini, "Analysis and Simulation of a Digital Mobile channel Using Orthogonal Frequency Division Multiplexing," IEEE Trans. on Communications, vol. 33, pp. 665-675, Jul. 1985.

[3] H. Rohling, T. May, K. Bruninghaus, and R. Grunheid, "Broadband OFDM radio transmission for multimedia applications," in Proc. the IEEE, vol. 87, pp. 1778-1789, Oct. 1999.

[4] X. D. Li and L. J. Cimini, "Effects of clipping and filtering on the performance of OFDM," in Proc. 1997 IEEE $47^{\text {th }}$ Vehicular Technology Conf. 1997, vol. 3, pp. 1634-1638.

[5] X. D. Li and L. J. Cimini, "Effects of clipping and filtering on the performance of OFDM," IEEE Communication Letters, vol. 2, pp. 131-133, May 1998.

[6] J. Armstrong, "Peak-to-average power reduction for OFDM by repeated clipping and frequency domain filtering," Electronics Letters, vol. 38, 246-47, Feb. 2002.

[7] F. Ono and H. Imai, "Performance analysis of deliberately clipped OFDM signals," IEEE Trans. on Communications, vol. 50, pp. 89-101, Jan. 2002.

[8] J. A. Davis and J. Jedwab, "Peak-to-mean power control and error correction for OFDM transmission using Golay sequences and Reed-Muller codes," Electronics Letters, vol. 45, 267-268, Feb. 1997.

[9] P. Mukunthan and P. Dananjayan. (December 2010). Modified PTS with FECs for PAPR Reduction of OFDM Signals. Intl. Journal of Computer Applications [Online]. 11(3). pp. 38-43. Available: http://www.ijcaonline.org/archives/volume11/number3/1559-2081.
[10] H. Breiling, S. H. Muller-Weinfurtner, and J. B. Huber, "SLM peak-power reduction without explicit side information," IEEE Communication Letters, vol. 5, 239-241, June 2001

[11] L. Yang, K. K. Soo, S. Q. Li, and Y. M. Siu, "PAPR reduction using low complexity PTS to construct of OFDM signals without side information," IEEE Trans. on Broadcasting, vol. 57, pp. 284-290, June 2011.

[12] S. G. Kang, J. G. Kim, and E. K. Joo, "A novel subblock partition scheme for partial transmit sequence OFDM," IEEE Trans. on Broadcasting, vol. 45, pp. 333-338, Sep. 1999.

[13] S. H. Muller and J. B. Huber, "OFDM with reduced peak-to-average power ratio by optimum combination of partial transmit sequences," Electronics Letters, vol. 33, pp. 368-369, Feb. 1997.

[14] T. Ya-fei, D. Rong-hua, Y. Xiao-an, and T. Hai-wei, "PAPR reduction of OFDM signals using Modified Partial Transmit Sequences," in Proc. 2009 CISP'09 2 $2^{\text {nd }}$ International Congress, 2009, pp. 1-4.

[15] X. G. Doukopoulos and G. V. Moustakides, "Blind adaptive channel estimation in OFDM systems," IEEE Trans. on Wireless Communications, vol. 5, pp. 1716-1725, July 2006.

[16] J. J. Van de Beek, O. Edfors, M. Sandell, S. K. Wilson, and P. O. Borjesson, "On channel estimation in OFDM systems," in Proc. 1995 45 ${ }^{\text {th }}$ Vehicular Technology IEEE Conf., 1995, pp. 814-819.

[17] S. Wu and Y. Bar-Ness, "OFDM Channel Estimation in the presence of frequency offset and phase noise," in Proc.2003 ICC'03 IEEE Conf. 2003, pp. 3366-70

[18] S. Coleri, M. Ergen, A. Puri, and A. Bahai, "Channel estimation techniques based on pilot arrangement in OFDM systems," IEEE Trans. on Broadcasting, vol. 48, pp. 223-229, Sep. 2002.

[19] M. Hsieh and C. Wei, "Channel estimation for OFDM systems based on comb-type pilot arrangement in frequency selective fading channels," IEEE Trans. on Consumer Electronics, vol. 44, pp. 21-225, Feb. 1998

[20] T. M. Ma, Y. S. Shi, and Y. G. Wang, "A Low complexity MMSE for OFDM systems over frequency-selective fading channels," IEEE Communication Letters, vol. 16, pp. 304-306, Mar. 2012.

[21] M. Thanigasalam and P. Dananjayan, "BER Analysis of OFDM receiver using LS Channel estimation method with modified PTS for different subblocks," in Proc. EAS 2012 IASTED Conf., 2012, pp 432-436.

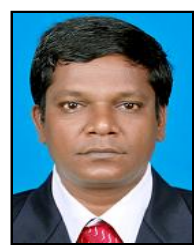

M. Thanigasalam received B.E. degree in Electronics \& Communication Engineering from Bharathiar University, Coimbatore, India in 1990 and M. Tech. degree in Electronics and Communication Engineering from Pondicherry Engineering College, Pondicherry, India in 1994. He has nineteen years of teaching experience. He is currently working as HOD in the Department of Electronics and Communication Engineering, Karaikal Polytechnic College, Karaikal, India. He is a part time Research Scholar in the Department of Electronics and Communication Engineering, Pondicherry Engineering College, Pondicherry, India. His research interests include computer networks and wireless communication.

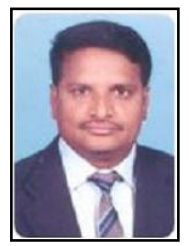

P. Dananjayan received Bachelor of Science from University of Madras in 1979, Bachelor of Technology in 1982 and Master of Engineering in 1984 from the Madras Institute of Technology, Chennai and Ph.D. degree from Anna University, Chennai in 1998. He is working as Professor in the Department of Electronics and Communication Engineering, Pondicherry Engineering College, Pondicherry, India. He has been as visiting professor to AIT, Bangkok. He has to his credit more than 90 publications in National and International Journals. He has presented more than 160 papers in National and International Conferences. He has guided $11 \mathrm{Ph} . \mathrm{D}$. candidates and is currently guiding 7 Ph.D. students. His research interests include spread spectrum techniques, wireless communication, wireless adhoc and sensor networks. 